\title{
The Advantages of Nanotechnology in Medical Field
}

\author{
Abdullah Alshahrani \\ University of Bridgeport, Electrical Engineering Department, Bridgeport, CT USA
}

\begin{abstract}
The paper evaluates the advantage of Nanotechnology in medical field. These advantages are in four points; in each point I highlighted the important applications and opportunities for these advantages in Nano medicine. This model could be used as a guideline for other researches, which is related to same topic. Also, this model can represent the effective side of Nano technology in medical field. This model highlighted the important advantages for nanotechnology in medical filed. Also; the model adds value for this area of study.
\end{abstract}

Keywords: Nanotechnology, repairing tissues, early detection, drug delivery.

\section{INTRODUCTION}

As we know that now our lives are depending on technology for everything around us. With technology everything was difficult in the past become easy now (Harris, 2004) .It is hard to confine technology in one side .Actually, it has many sections and types in every field(Mostafapour, Rezaei, \& Ghabousi, 2012) .We know that nanotechnology is one of the important technologies in this world. It has many applications in many different fields such as energy, Nano devices, Nano fabrics, and Nano bio technology (Corbett, McKeown, Peggs, \& Whatmore, 2000). In fact, Nano medicine is one of the important nanotechnology applications (Murday, Siegel, Stein, \& Wright, 2009) and (M. Chen, Lin, \& Cheng, 2013) . It has many applications in medical field such as, nanomaterial, Nano electronic biosensors, and molecular nanotechnology, and drug delivery (K. Jain, 2011) . Even though Nano medicine's researches and products are in early stage, but it achieved huge success in medical field with increasing the number of diseases and the difficulties to determine and treat them (Papageorgiou, Savvides, \& Zachariadis, 2007). This success for Nano medicine lays in the ways and devices, which are, relies on this technique. Now, there are many projects and studies are depending on Nano technology; also, many countries devote their scientists to develop this technology because it will increase the rate of their economy (Dressler \& Kargl, 2012) and (P. Smith, 2007) .In this papers I will highlight the advantages for Nano medicine which are the methods to detect disease in early stage, the small size of Nano medicine devises, repairing tissues inside the body, and drug delivery system. Those advantages added a great success in medical field. Nano medicine helped both doctors and patients to accelerate progress in medicine forward. Even though this technology has side of effect as any technology but in this research I focused on its advantages regardless on its effect; this is because Nano medicine proved it effectiveness in medical field as its effectiveness in other fields too.

Recently combining research methods (Gable, 1994) p. 112 became increasingly important in particular when pursuing the goal of gaining "rich theoretical insights (Dyer \& Wilkins, 1991) p. 613." One form for "developing new theoretical insights" (LePine \& WilcoxKing, 2010) p. 506 is the review centric research approach in which a researcher reviews "existing theory and research" (LePine \& Wilcox-King, 2010) p. 506, but the argument can be made that we also can include case study research findings that are based on the real world observation of practitioners and organizations e.g. (Eisenhardt, 1989), (Dyer \& Wilkins, 1991). In the research presented here the focus is on combining the most important "previously established studies and concepts" that I have identified in the academic literature based on which I provide a synthesis that "advances our understanding" (LePine \& Wilcox-King, 2010) p. 507.” In this study I identify the most important success factors that help in the current business or organizational situation to build our goal to accomplish a competitive advantage.

the model which was used in this study to express the importance of the actions in our organization ,contains the goal which focused on the fundamental purpose for using nanotechnology in medical field. In my research I have deeply expressed the evaluation and applications of this technology. Also, I have highlighted the specific advantages and applications, which are already applied in medical field. It is my argument that there is a need to intensification the researches and studies in certain medical areas by using nanotechnology to enhance the quality and effectiveness for this field.

\section{THE ADVANTAGES OF NANOTECHNOLOGY IN THE MEDICAL FIELD}

As a definition for Nano medicine is that Nano medicine consider as the repair, control, construction and monitoring at the molecular level of human biological systems by using engineered Nano devices and nanostructures (Morrow, Bawa, \& Wei, 2007) .Nano technology in medical field is used to quantify best clinically symptoms and outcomes, such as pain, and diseases, which were difficult to measure. In addition, the applications of nanotechnology in medicine create a great opportunity to improve the quality of medical and health care; also, increased knowledge and researches of modern medicine with the capacity to manipulate with materials at 
the Nano scale to accurate diagnosis and therapy, with are small enough to reach every single cell in human observance regulatory and safety issues (Gabellieri \& body(Hogg, Freitas, \& Robert, 2012). There are two ways Frima, 2011). Recently, there is a new strategy for to design Nano devices. These ways are bottom-up method improving blood compatibility. This strategy lies in design and top-down method. The last one depends on and fabrication of Nano scale on biological tissues or arrangement atom-by-atom or molecule-by-molecule. This biomaterial surfaces (Chen, Han, \& Jiang, 2011). Also, way is used to design medicine devices. Top-Down Nano-bio composites show properties with preservation of Method is used to design smaller devices by using larger the material biodegradability without eco-toxicity, that devices to assemble them (Asiyanbola \& Soboyejo, resulting is a great promising to use in biomedical 2008).The greatest properties for Nano medicine devices applications (Bordes, Pollet, \& Avérous 2009). Scientists are the small size which let those devices to inter the in tissue engineering dedicated their research in human body in which the traditional medicine devices nanotechnology, specifically Nano fibers; which is cannot. Like Surgical Nano robots as an example for considering as a way to develop tissue engineering medicine devices could be introduced into the human body scaffolds and repair damaged tissue. (Barnes, Sell, Boland, Simpson, \& Bowlin, 2007). As an example, Nano fiber is used as channel to connect the nerve gap with Peripheral nerve without any atrophy .thus, implant artificial nerve is integrated with host nerve as proof for that is the smooth transition of both connection zones (Zhan et al., 2013) . There are clinical applications of tissue engineering to implant nerves and repair of damaged nerves. This application is based on using freeze-dried Nano-silver-collagen to use as scaffold material to assimilate nerve regeneration (Ding, Luo, Zheng, Hu, \& Ye, 2010). Also, using peptide Nano fiber scaffold to treat injured brain is a promising approach for the reconstruction for this kind of tissues (Leung, Wang, \& $\mathrm{Wu}, 2012$ ). In the United States there are around seven million people each year suffering from Bone fracture, and musculoskeletal. The clinical treatment cost around \$215 billion annually (Xu et al., 2012). The great success for bone graft by using Nano fiber technology is that insurance a synthetic source of bone tissue. This success proves the ability to produce Nano fibers by use electro spinning process for incorporating an organ soluble, nontoxic fatty acid salt (Ruckh, Kumar, Kipper, \& Popat, 2010) . In the United States, there are around 60,240 people are suffering from urinary bladder cancer. In medical field there are many methods to treat bladder cancer and other organ that has cancer such as partial resection the diseased part, chemotherapy, and radiation. But these ways will impact the patients in many sides. But with Nano technology in medical there is a method to treat bladder cancer to replacement bladder tissue by Threedimensional, Nano-structured PLGA scaffolds (Pattison, Wurster, Webster, \& Haberstroh, 2005). In addition, The development of nanotechnology of vascular tissue bio fabrication is one of important technological in tissue engineering (Mironov, Kasyanov, \& Markwald, 2008). Nano fibrous scaffolds can mimic collagen, which is natural polymer that provides opportunities in tissue engineering field. (Smith \& Ma, 2004). Tissue engineering and regenerative medicine aims to insurance and develop biological substitutes which can be used to restore or improve or replace damaged tissue and organ .The success for nanotechnology in medical field helps to treat damaged tissue of bone, cartilage, vascular, and neural. (Zhang \& Webster, 2009).

The development is in medicine devices is that using in vivo. Nano technology can make revolution in diagnostic and health care by Nano medicine devices. Those devices cavities in the body. This devices is controlled by a surgeon (Freitas \& Robert, 2005a) and (Khawaja, 2011). There are many applications for Nano medicine device but the important application is nanotechnology on a chip (Freitas \& Robert, 2005b) . It is designed to determine or analyze DNA and proteins between many other categories. The NPs also design to act as marker for stem cells, bacteria, and viruses to act as tracking devices too (Allison, Mota, Bagnato, \& Sibata, 2008). The advantage of Nano tools is their sizes which are like molecules which let clinicians to treat every cell in the body. These Nano tools made of biodegradable and biocompatible nanomaterial. Using strategies for specific targeting is preventing the harm. Also, biosensors can control drug delivery and individual cell. It also used to repair existing cells instead of destroy diseased cells. Even though Nano medical tools have tiny size, but have strong impact on medicine and health care figure 1. Sensitive and early diagnosis will lead to detect and treat diseases in early stage. (Leary \& James, 2010).

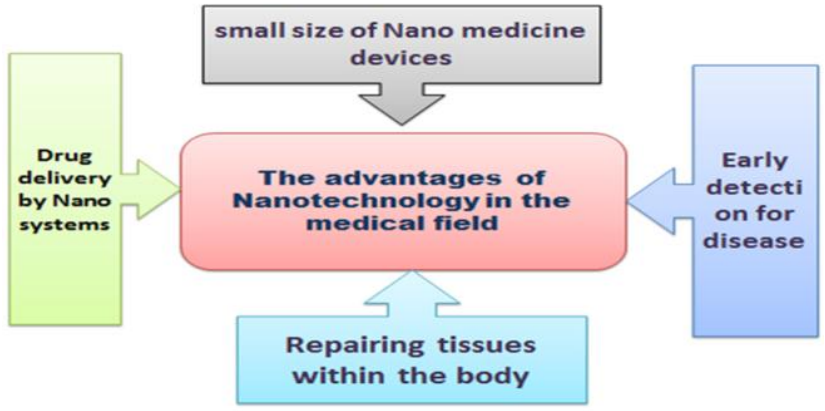

Figure1: Model for the Advantages of Nanotechnology in Medical Field

\section{DRUG DELIVERY BY NANO SYSTEMS}

The new drug carriers in Nano-scale offer the chance of development and increase the therapeutic index of drug molecules. This increasing is proved by their effectiveness, decrease their toxicity for physiological tissues (Kontogiannopoulos et al., 2012) .The approaches of drug delivery are of importance for medicine and healthcare. Materials and nanotechnology play an important role to make advancement of drug delivery(Zhang, Chan, \& Leong, 2013). Drug delivery requires that accurate navigation through biological processes in the body by using carriers which carry the drug; so, these new opportunities will achieve more 
success when properties for particles can be controlled in traditional ultrasound (Wadajkar et al.). Some of real time. With these advantages we can timely control the researchers devoted their researches on the applications of particles by their interactions with other cells. Also control size, shape, and surface chemistry of these particles. (Yoo, Doshi, \& Mitragotri, 2011). As an example for drug delivery by Nano system is Lithographic. There are new methods of drug delivery devices, so Lithography is one of fabrication strategy to offer smart drug delivery systems (Randall, Leong, Bassik, \& Gracias, 2007). It is one of drug delivery methods which offer a group of tools that allow substrates at the nanometer to millimeter scale; it allows microelectronic devices and micro electro mechanical systems to be fabricated in big numbers on silicon ship substrates. The important strategy for drug delivery is that peptide therapeutic delivery to brain. This strategy based on use chimeric peptides. (Pardridge, 1995). The field of drugs delivery with nanoparticles achieved that a better understanding of nanoparticle interactions are a proof for the success of this field. Over the last five years the number of nanoparticle skin delivery research has dramatically risen making this a promising area of science (Prow et al., 2011) . Micro Nano scale technologies such as lithographic techniques and microfluidic represent promising methods to develop the fields of tissue engineering, drug discovery, and diagnostics. Micro fabrication techniques provide drug delivery applications which are important in medical field (Sant et al., 2012).

\section{EARLY DETECTION FOR DISEASE}

The main goal of Nano technology in medical field is that accurate diagnose and early detection for diseases to effective treatment without side effects. Also evaluate the effective treatment by non-invasively methods(Caruthers, Wickline, \& Lanza, 2007). There is no doubt that nanotechnology become cornerstone in medical field. There are many applications for nanotechnology in cancer for fight pain, suffering and death. There are modalities already are in use for diagnostic and treatment, with many others at different stages of pre-clinical testing. Nanotechnology has been used for improving ovarian cancer diagnosis and treatment also, it used to early diagnosis imaging and screening of ovarian cancer (PopeHarman, Cheng, Robertson, Sakamoto, \& Ferrari, 2007). This technology has already applied in diagnosis and treatment of diseases; so its applications in the field of medicine achieved huge success. By Nano technology we could prevent dental caries by antimicrobial nanoparticles. When used fillers and toothpastes with Silver particles as antibacterial agents, it will prevent bacterial to grow and tooth decay. Also, by Nanotechnology clinicians can diagnose and treat oral malignancies at early stage (Subramani \& Ahmed, 2012). Recent developments on Nano technology have provided some hope to detect and treat the Deep vein thrombosis. The researches for micro technology, which related to the diagnosis and therapy of cardiovascular diseases, proved that Nanoparticle systems able to both diagnose and treat the thrombus. In addition, Nano-diagnostic systems can also travel to a large area within the organ, so that allow to visualization through a larger area. This advantage does not available for proteomics which used to detect disease and candidate biomarkers for early diagnosis. In addition, the important diagnostic applications are gold nanoparticles, carbon nanotubes and silicon nanowires. (Ray, Reddy, Choudhary, Raghu, \& Srivastava, 2011).The one of main reasons for developing glucose sensors in vivo is detection of hypoglycemia in individuals who suffering with insulin dependent diabetes. Fluorescent micro/Nano scale devices for glucose sensing become possible to use in dermis which allow transdermal monitoring the rates of glucose changes in the body (K. K. Jain, 2005).

Nanotechnology achieved a great successful in many fields. But still the medical field is the important because year by year there are many diseases appear; so, the medicine methods must keep pace with this growing. Also, the traditional method and devices, which were used to treat patients and deduct the diseases, are tiring and stressful for both the doctor and patient. Nano technology with its properties could help the medical field to become towered. Those properties lay in four sides, which are the small size for Nano medicine devices, early detection for diseases, drug delivery methods, and repairing tissues within the body.

The First advantage, which is small size for medicine devices, achieved huge success in medical field. By this advantage the treatment and detect the diseases become easier than using traditional devisees, which have big sizes. Also, those devisees with small sizes facilitated surgical procedure. The surgeons can controlled those devices inside patient's bodies. On the other hand those devices proved its effectiveness in vitro by small devices, which called lap on ship. This lap on ship works as laboratory. It is important to confirm that the small size for Nano medicine devises proved their success in both vivo and vitro.

The second advantage, which is drug delivery by Nano systems, is important way to target diseased cells in the body. The best example for this technique is that targeting cancer cells without impact the normal cells. Actually, this technique proved successful result in this field. In fact, nanotechnology lies in the carrier for this drug. In other word the drug will be carried on different Nano scales, which have small sizes to inter and target the diseased cells.

The third advantage which is the early detection for disease this advantage allow the doctors to detect the diseases in early stage. So, the benefit from this advantage will provide more easy treatment for the diseases than detect them in later stage. In fact, this advantage lies in Nanoparticle, which is used to diagnose and detect the disease in early stage.

Fourth advantage, which is repairing tissue inside the body, is important advantage for sensitive tissues. Nanotechnology has the ability to repair tissues within the body with Nano fiber. In fact, the great develop of tissue engineering is scaffolds which are used to repair damaged tissue. For neural tissue engineering the Sub-micron and 
Nano-scale fibrous scaffolds that mimic natural cell can repair the damage in those sensitive tissues.

\section{CONCLUSION}

This research was about the advantages for Nano technology in medical field. In fact there is no doubt that the great benefits for this technique are in many fields. But in this research I focused on nanotechnology advantages in medical. Those advantages lie in four points; the first point is the small size for Nano medicine devices, which can enter the body and reach the specific tissue. Also those devices can be in vivo such as lab on ship. Nano medicine devises proved its success in both vivo and vitro. The second point that is the drug delivery systems achieved a great stage for treat the censer diseases. Drug delivery systems with small size fore nonlocals allow the drug to treat and control the diseased cells. Even though the researches for this technique are in early stage but it proved its effectiveness. The third point is early detection; Nano technology has the ability to diagnose and detect the disease in early stage. These advantages play an important role to detect and treat the censer disease in early stage also, for other types of diseases. The fourth point is repairing tissue within the body, which is adding to medical field great success. By Nano fiber the doctors can repair the damage tissue which are caused for example by injuries, burns, or modify some damage tissue after healing form diseases.

Even though Nano technology has advantages in many fields, it also has side of effects as any technology. But we can regardless those effects for the sake of benefit of its advantages. That is what I did in this research. I focused and highlighted the important side for this technology in medical field. Also, it is difficult that to control the both sides in one research, this will underestimate the value of its success. In the end of this paper we have to expect that after some years Nano technology will solve a lot of difficulties and challenges in medical field.

\section{REFERENCES}

[1] Allison, R., Mota, H., Bagnato, V., \& Sibata, C. (2008). Bionanotechnology and photodynamic therapy-State of the art review. Photodiagnosis and Photodynamic Therapy, 5(1), 19-28.

[2] Asiyanbola, B., \& Soboyejo, W. (2008). For the Surgeon: An Introduction to Nanotechnology. Journal of Surgical Education, 65(2), 155-161

[3] Allison, R., Mota, H., Bagnato, V., \& Sibata, C. (2008). Bionanotechnology and photodynamic therapy-State of the art review. Photodiagnosis and Photodynamic Therapy, 5(1), 19-28.

[4] 2. Asiyanbola, B., \& Soboyejo, W. (2008). For the Surgeon: An Introduction to Nanotechnology. Journal of Surgical Education, 65(2), 155-161.

[5] 3. Barnes, Sell, Boland, Simpson, \& Bowlin. (2007). Nanofiber technology: Designing the next generation of tissue engineering scaffolds. Advanced Drug Delivery Reviews, 59(14), 1413-1433.

[6] 4. Bordes , P., Pollet , E., \& Avérous , L. (2009). Nanobiocomposites: Biodegradable polyester/nanoclay systems. Progress in Polymer Science, 34(2), 125-155.

[7] 5. Caruthers, D., Wickline, A., \& Lanza, M. (2007) Nanotechnological applications in medicine. Current Opinion in Biotechnology, 18(1), 26-30.

[8] 6. Chen, Han, \& Jiang. (2011). On improving blood compatibility: From bioinspired to synthetic design and fabrication of biointerfacial topography at micro/nano scales. Colloids and Surfaces B: Biointerfaces, 85(1), 2-7.
[9] 7. Chen, M., Lin, Y., \& Cheng, T. (2013). Public attitudes toward nanotechnology applications in Taiwan. Technovation, 33(2-3), 88-96

[10] 8. Corbett, J., McKeown, P., Peggs, G., \& Whatmore, R. (2000). Nanotechnology: International Developments and Emerging Products. CIRP Annals - Manufacturing Technology, 49(2), 523545.

[11] 9. Ding, Luo, Zheng, Hu, \& Ye. (2010). Rapid repair and regeneration of damaged rabbit sciatic nerves by tissue-engineered scaffold made from nano-silver and collagen type I. Injury, 41(5), 522-527.

[12] 10. Dressler, F., \& Kargl, F. (2012). Towards security in nanocommunication: Challenges and opportunities. Nano Communication Networks, 3(3), 151-160

[13] 11. Dyer, G. W. J., \& Wilkins, A. L. (1991). Better stories, not better constructs, to generate better theory: A rejoinder to Eisenhardt. Academy of Management Review, 16(3), 613-619.

[14] 12. Eisenhardt, K. M. (1989). Building Theories from case study research. Academy of Management Review, 14(4), 532-550.

[15] 13. Freitas, \& Robert. (2005a). Nanotechnology, nanomedicine and nanosurgery. International Journal of Surgery, 3(4), 243-246.

[16] 14. Freitas, \& Robert. (2005b). What Is Nanomedicine? Diseasea-Month, 51(6), 325-341.

[17] 15. Gabellieri, C., \& Frima, H. (2011). Nanomedicine in the European Commission policy for nanotechnology. Nanomedicine: Nanotechnology, Biology and Medicine, 7(5), 519-520.

[18] 16. Gable, G. G. (1994). Integrating Case Study and Survey Research Methods: An Example in Information Systems. European Journal of Information Systems, 3(2), 112-126.

[19] 17. Harris, D. (2004). Evaluating the transfer of technology between application domains: a critical evaluation of the human component in the system. Technology in Society, 26(4), 551-565.

[20] 18. Hogg, T., Freitas, \& Robert. (2012). Acoustic communication for medical nanorobots. Nano Communication Networks, 3(2), 83102.

[21] 19. Jain, K. (2011). 1.45 - Nanobiotechnology. In M.-Y. Editorin-Chief: Murray (Ed.), Comprehensive Biotechnology (Second Edition) (pp. 599-614). Burlington: Academic Press.

22] 20. Jain, K. K. (2005). Nanotechnology in clinical laboratory diagnostics. Clinica Chimica Acta, 358(1-2), 37-54.

[23] 21. Khawaja, A. (2011). The legacy of nanotechnology: Revolution and prospects in neurosurgery. International Journal of Surgery, 9(8), 608-614

[24] 22. Kontogiannopoulos, Assimopoulou, Hatziantoniou, Karatasos, Demetzos, \& Papageorgiou. (2012). Chimeric advanced drug delivery nano systems (chi-aDDnSs) for shikonin combining dendritic and liposomal technology. International Journal of Pharmaceutics, 422(1-2), 381-389.

[25] 23. Leary, \& James. (2010). Nanotechnology: what is it and why is small so big? Canadian Journal of Ophthalmology / Journal Canadien d'Ophtalmologie, 45(5), 449-456.

[26] 24. LePine, J. A., \& Wilcox-King, A. (2010). Developing novel theoretical insight from reviews of existing theory and research. Academy of Management Review, 35(4), 506-509.

[27] 25. Leung, Wang, \& Wu. (2012). Chapter nine - Peptide Nanofiber Scaffold for Brain Tissue Reconstruction. In D. Nejat (Ed.), Methods in Enzymology (Vol. Volume 508, pp. 177-190): Academic Press.

[28] 26. Mironov, Kasyanov, \& Markwald. (2008). Nanotechnology in vascular tissue engineering: from nanoscaffolding towards rapid vessel biofabrication. Trends in Biotechnology, 26(6), 338-344.

[29] 27. Morrow, J., Bawa, R., \& Wei, C. (2007). Recent Advances in Basic and Clinical Nanomedicine. Medical Clinics of North America, 91(5), 805-843.

[30] 28. Mostafapour, M., Rezaei, H., \& Ghabousi, S. (2012). The application of information technology and its role on entreprenesurs success. Procedia Technology, 1(0), 98-101.

[31] 29. Murday, S., Siegel, W., Stein, J., \& Wright, J. (2009). Translational nanomedicine: status assessment and opportunities. 